\title{
Article
}

\section{Directional Shift-Stable Functions}

\author{
Radko Mesiar 1,2,+(iD) and Andrea Stupňanová 1,*,+(D) \\ 1 Faculty of Civil Engineering, Slovakia University of Technology in Bratislava, Radlinského 11, \\ 81005 Bratislava, Slovakia; radko.mesiar@stuba.sk \\ 2 Department of Algebra and Geometry, Faculty of Science, Palacky University Olomouc, 17 Listopadu 12, \\ 77146 Olomouc, Czech Republic \\ * Correspondence: andrea.stupnanova@stuba.sk \\ + Both authors contributed equally to this work.
}

check for

updates

Citation: Mesiar, R.; Stupňanová, A. Directional Shift-Stable Functions. Mathematics 2021, 9, 1077.

https://doi.org/10.3390/math9101077

Academic Editor: Daniel Gómez Gonzalez

Received: 7 April 2021

Accepted: 5 May 2021

Published: 11 May 2021

Publisher's Note: MDPI stays neutral with regard to jurisdictional claims in published maps and institutional affiliations.

Copyright: () 2021 by the authors. Licensee MDPI, Basel, Switzerland. This article is an open access article distributed under the terms and conditions of the Creative Commons Attribution (CC BY) license (https:// creativecommons.org/licenses/by/ $4.0 /)$.

\begin{abstract}
Recently, some new types of monotonicity-in particular, weak monotonicity and directional monotonicity of an $n$-ary real function-were introduced and successfully applied. Inspired by these generalizations of monotonicity, we introduce a new notion for $n$-ary functions acting on $[0,1]^{n}$, namely, the directional shift stability. This new property extends the standard shift invariantness (difference scale invariantness), which can be seen as a particular directional shift stability. The newly proposed property can also be seen as a particular kind of local linearity. Several examples and a complete characterization for the case of $n=2$ of directionally shift-stable aggregation and pre-aggregation functions are also given.
\end{abstract}

Keywords: aggregation function; directional monotonicity; pre-aggregation function; shift invariantness

\section{Introduction}

The theory of aggregation functions and related functions is an explosively growing branch of mathematics. This fact is directly related to the increase in computer-based applications in fields such as multicriteria decision support, fuzzy-rule-based systems, fuzzy logic, and image processing. For more details, see books [1-5]. Recall that the most considered framework for aggregation functions deals with the domain $[0,1]^{n}, n \in \mathbb{N}$, i.e., with the input $n$-tuples from $[0,1]^{n}$, and with the co-domain $[0,1]$ for outputs, boundary conditions $A(0, \ldots, 0)=0$ and $A(1, \ldots, 1)=1$, and the monotonicity of $A$. We denote $\mathcal{A}_{n}$ as the class of all such $n$-ary aggregation functions.

Recently, inspired by potential applications, several modifications of aggregation functions have been considered. In particular, Wilkin and Beliakov [6] have proposed the concept of weak aggregation functions, where the monotonicity of aggregation functions was replaced by the weak monotonicity, i.e.,

$$
A\left(x_{1}+c, \ldots, x_{n}+c\right) \geq A\left(x_{1}, \ldots, x_{n}\right)
$$

for any $c>0$ and $\left(x_{1}, \ldots, x_{n}\right) \in[0,1]^{n}$ such that $\left(x_{1}+c, \ldots, x_{n}+c\right) \in[0,1]^{n}$. As a typical weak aggregation function, we recall the well-known (minimal and maximal) modal value from statistics. We denote by $\mathcal{W}_{n}$ the class of all $n$-ary weak aggregation functions.

Weak monotonicity is a particular case of the directional monotonicity [7]. For a vector $\vec{r}=\left(r_{1}, \ldots, r_{n}\right)$, a function $F:[0,1]^{n} \rightarrow \mathbb{R}$ is $\vec{r}$-increasing (increasing in direction $\vec{r}$ ) if

$$
F(\mathbf{x}+c \vec{r}) \geq F(\mathbf{x})
$$

for any $c>0$ and $\mathbf{x} \in[0,1]^{n}$ such that $\mathbf{x}+c \vec{r} \in[0,1]^{n}$. Clearly, weakly increasing functions are simply those that are $(1, \ldots, 1)$-increasing. Lucca et al. [8] proposed the concept of preaggregation functions, i.e., functions $F:[0,1]^{n} \rightarrow[0,1]$ satisfying the boundary conditions for aggregation functions, where the monotonicity of $F$ is considered to be $\vec{r}$-increasingness 
with respect to some direction $\vec{r} \in\left[0, \infty\left[{ }^{n} \backslash\{(0, \ldots, 0)\}\right.\right.$. We denote by $\mathcal{P}_{n}$ the class of all $n$-ary pre-aggregation functions.

In addition, some other classes of $n$-ary real functions are considered in this paper, namely

- $\quad$ The class $\mathcal{F}_{n}$ of all $n$-ary fusion functions [7], i.e., functions $F:[0,1]^{n} \rightarrow[0,1]$;

- The class $\mathcal{B}_{n}$ of all $n$-ary bounded functions on $[0,1]^{n}$, i.e., bounded functions $F$ : $[0,1]^{n} \rightarrow \mathbb{R}$;

- $\quad$ The class $\mathcal{S}_{n}$ of all $n$-ary semi-aggregation functions, i.e., fusion functions satisfying the boundary conditions for aggregation functions.

The next inclusions are obvious:

$$
\mathcal{B}_{n} \supset \mathcal{F}_{n} \supset \mathcal{S}_{n} \supset \mathcal{P}_{n} \supset \mathcal{W}_{n} \supset \mathcal{A}_{n}
$$

(If $n>1$, all inclusions are strict; if $n=1$, then $\mathcal{P}_{1}=\mathcal{W}_{1}=\mathcal{A}_{1}$.)

One of the most important possible properties of (aggregation) functions is their shift invariance characterized by

$$
F\left(x_{1}+c, \ldots, x_{n}+c\right)=F\left(x_{1}, \ldots, x_{n}\right)+c
$$

for any real constant $c$ (it is enough to consider $c>0$ ) and $\left(x_{1}, \ldots, x_{n}\right) \in[0,1]^{n}$ such that $\left(x_{1}+c, \ldots, x_{n}+c\right) \in[0,1]^{n}$. Obviously, the shift invariance (also called the difference scale invariance in measurement theory) ensures weak monotonicity.

The aim of this paper is a generalized look at shift stability as inspired by the way the directional monotonicity generalizes the weak monotonicity. Considering a fixed non-negative vector $\vec{r}=\left(r_{1}, \ldots, r_{n}\right)$, we propose and discuss (aggregation) functions with increments $F(\mathbf{x}+c . \vec{r})-F(\mathbf{x})$ solely dependent on the real constant $c$, independent of the input n-tuple $\mathbf{x}$. Clearly, such functions are then necessarily $\vec{r}$-directionally monotone. Subsequently, we expect to see the applications of our results in fuzzy classification, image processing, and all other fields where directionally monotone functions are successfully applied.

The paper is organized as follows: In the next section, we introduce the notion of directional shift stability for the above-discussed functional classes and give some basic examples. In Section 3, we recall some known characterizations and add some new characterizations of binary shift-invariant functions from the above-introduced classes. Section 4 deals with general binary directional shift-stable functions. Finally, some concluding remarks are presented.

\section{Directional Shift-Stable Functions}

The following property of functions was inspired by shift invariantness and directional monotonicity.

Definition 1. Let $F \in \mathcal{B}_{n}$ and $\vec{r} \in \mathbb{R}^{n}$. Function $F$ is deemed $\vec{r}$-directional shift stable whenever

$$
F(\mathbf{x}+c \vec{r})-F(\mathbf{x})=F(\mathbf{y}+c \vec{r})-F(\mathbf{y})
$$

for all $\mathbf{x}, \mathbf{y} \in[0,1]^{n}$ and $c \in \mathbb{R}$ such that $\mathbf{x}+c \vec{r}, \mathbf{y}+c \vec{r} \in[0,1]^{n}$.

Example 1. Define $F:[0,1]^{3} \rightarrow \mathbb{R}$ by

$$
F\left(x_{1}, x_{2}, x_{3}\right)=\frac{x_{1}+x_{2}+x_{3}}{3}-\frac{\left(x_{1}-2 x_{2}\right)^{2}+\left(x_{1}-3 x_{3}\right)^{2}+\left(2 x_{2}-3 x_{3}\right)^{2}}{60} .
$$


Obviously, $F \in \mathcal{B}_{3}$ (moreover, it can be shown that $F \in \mathcal{F}_{3} \backslash \mathcal{P}_{3}$ ). Let $\vec{r}=\left(1, \frac{1}{2}, \frac{1}{3}\right)$. Then, for any $\mathbf{x} \in[0,1]^{3}$ and $c \in \mathbb{R}$ such that $\mathbf{x}+c \vec{r} \in[0,1]^{3}$, it holds

$$
\begin{aligned}
& F(\mathbf{x}+c \vec{r})-F(\mathbf{x}) \\
= & \left(\frac{x_{1}+c+x_{2}+\frac{c}{2}+x_{3}+\frac{c}{3}}{3}-\frac{\left(x_{1}-2 x_{2}\right)^{2}+\left(x_{1}-3 x_{3}\right)^{2}+\left(2 x_{2}-3 x_{3}\right)^{2}}{60}\right) \\
& -\left(\frac{x_{1}+x_{2}+x_{3}}{3}-\frac{\left(x_{1}-2 x_{2}\right)^{2}+\left(x_{1}-3 x_{3}\right)^{2}+\left(2 x_{2}-3 x_{3}\right)^{2}}{60}\right) \\
= & \frac{11}{18} c,
\end{aligned}
$$

independently of $\mathbf{x}$, and, hence, $F$ is $\vec{r}$-directional shift-stable.

The next result brings an alternative view on the directional shift stability, which, in several cases, simplifies the study of directional shift-stable functions.

Theorem 1. Let $F \in \mathcal{B}_{n}$ be an $\vec{r}$-directional shift-stable function, where $\vec{r} \in \mathbb{R}^{n}$. Then, and only then, there is a real constant $k$ such that for each $\mathbf{x} \in[0,1]^{n}$ and $c \in \mathbb{R}$ satisfying $\mathbf{x}+c \vec{r} \in[0,1]^{n}$ it holds

$$
F(\mathbf{x}+c \vec{r})=F(\mathbf{x})+k c .
$$

Proof. The sufficiency is obvious as, then, $F(\mathbf{x}+c \vec{r})-F(\mathbf{x})=k c$ does not depend on $\mathbf{x}$. Consider that $F$ is $\vec{r}$-directional shift stable. The difference $F(\mathbf{x}+c \vec{r})-F(\mathbf{x})$, if well defined, does not depend on $\mathbf{x}$. Denote this difference as $\varphi(c)$. Note also that the domain of possible constants $c$ is a closed subinterval $[a, b]$ containing 0 (e.g., if $\vec{r}=(1, \ldots, 1)$, then $c \in[-1,1]$ ). Hence, $\varphi:[a, b] \rightarrow \mathbb{R}$ is a bounded real function (this follows from the boundedness of $F)$. Moreover,

$$
\begin{aligned}
\varphi(c+d) & =F(\mathbf{x}+(c+d) \vec{r})-F(\mathbf{x}) \\
& =F((\mathbf{x}+c \vec{r})+d \vec{r})-F(\mathbf{x}+c \vec{r})+F(\mathbf{x}+c \vec{r})-F(\mathbf{x}) \\
& =\varphi(d)+\varphi(c)
\end{aligned}
$$

for any $c, d \in[a, b]$ such that also $c+d \in[a, b]$, i.e., $\varphi$ is an additive function. Hence, the classical Cauchy equation $\varphi(c+d)=\varphi(c)+\varphi(d)$, together with the boundedness of $\varphi$, means that there is some constant $k \in \mathbb{R}$ such that $\varphi(c)=k c$; see, e.g., [9]. Hence, the result $F(\mathbf{x}+c \vec{r})=F(\mathbf{x})+k c$ follows.

For simplicity, we introduce the notation for the $\vec{r}$-directional shift-stable function $F$ related to the constant $k$ by $F \stackrel{\text { s.s. }}{\sim}(\vec{r}, k)$. Directional shift stability ensures directional monotonicity, as formulated in the next result, which is a trivial consequence of Theorem 1.

Corollary 1. Let $\vec{r} \neq \overrightarrow{\mathbf{0}}$ and let $F \in \mathcal{B}_{n}$ be an $\vec{r}$-directional shift-stable function characterized by constant $k$, i.e., $F \stackrel{\text { s.s. }}{\sim}(\vec{r}, k)$. Then:

(i) If $k>0, F$ is $\vec{r}$-increasing;

(ii) If $k=0, F$ is $\vec{r}$-constant;

(iii) If $k<0, F$ is $\vec{r}$-decreasing.

Based on Theorem 1, we also see that if $F \in \mathcal{B}_{n}$ and $F \stackrel{\text { s.s. }}{\sim}(\vec{r}, k)$, then it is also $\alpha \vec{r}$ directional shift stable for any non-zero constant $\alpha$, and then $F$ is linked to the constant $\alpha \cdot k$, i.e., $F \stackrel{\text { s.s. }}{\sim}(\alpha \vec{r}, \alpha k)$,

$$
F(\mathbf{x}+c(\alpha \vec{r}))=F(\mathbf{x})+(\alpha \cdot k) c .
$$


Recall that for a function $F \in \mathcal{B}_{n}$, its dual $F^{d}:[0,1]^{n} \rightarrow \mathbb{R}$ is given by

$$
F^{d}\left(x_{1}, \ldots, x_{n}\right)=1-F\left(1-x_{1}, \ldots, 1-x_{n}\right) .
$$

Evidently, $F^{d} \in \mathcal{B}_{n}$. Moreover, the next result could be of interest.

Corollary 2. Let $F \in \mathcal{B}_{n}$ and $F \stackrel{\text { s.s. }}{\sim}(\vec{r}, k)$. Then, also $F^{d} \stackrel{\text { s.s. }}{\sim}(\vec{r}, k)$.

Proof. The result is a matter of a direct computation. Indeed, if $\mathbf{x}+c \vec{r} \in[0,1]^{n}$, then also $\mathbf{1}-(\mathbf{x}+c \vec{r})=(\mathbf{1}-\mathbf{x})-c \vec{r} \in[0,1]^{n}$. Then,

$$
\begin{array}{r}
F^{d}(\mathbf{x}+c \vec{r})=1-F((\mathbf{1}-\mathbf{x})-c \vec{r})=1-(F(\mathbf{1}-\mathbf{x})-k c) \\
=(1-F(\mathbf{1}-\mathbf{x}))+k c=F^{d}(\mathbf{x})+k c .
\end{array}
$$

Note also that

(i) if $F_{1}, \ldots, F_{m} \in \mathcal{B}_{n}$ and $F_{i} \stackrel{\text { s.s. }}{\sim}\left(\vec{r}, k_{i}\right)$ for $i=1, \ldots, m$, then also any linear combination $F=\sum_{i=1}^{m} \lambda_{i} F_{i}, \lambda_{1}, \ldots, \lambda_{m} \in \mathbb{R}$, belongs to $\mathcal{B}_{n}$, and it is $\vec{r}$-directional shift stable with constant $k=\sum_{i=1}^{m} \lambda_{i} k_{i}, F \stackrel{\text { s.s. }}{\sim}\left(\vec{r}, \sum_{i=1}^{m} \lambda_{i} k_{i}\right)$.

In particular, if $F \in \mathcal{B}_{n}$ and $F \stackrel{\text { s.s. }}{\sim}(\vec{r}, k)$, then $G=\frac{F+F^{d}}{2} \in \mathcal{B}_{n}$ is a self-dual function such that $G \stackrel{\text { s.s. }}{\sim}(\vec{r}, k)$;

(ii) if $F \in \mathcal{B}_{n}$ and $F \stackrel{\text { s.s. }}{\sim}\left(\vec{r}_{i}, k_{i}\right), i=1, \ldots, l$, then $F$ is also $\sum_{i=1}^{l} r_{i}$-directional shift stable with constant $k=\sum_{i=1}^{l} k_{i}, F \stackrel{\text { s.s. }}{\sim}\left(\sum_{i=1}^{l} r_{i}, \sum_{i=1}^{l} k_{i}\right)$;

(iii) if $F \in \mathcal{B}_{n}$ and $F \stackrel{\text { s.s. }}{\sim}(\vec{r}, k)$ and $\sigma:\{1, \ldots, n\} \rightarrow\{1, \ldots, n\}$ is a permutation, then

$$
F_{\sigma}\left(x_{1}, \ldots, x_{n}\right)=F\left(x_{\sigma(1)}, \ldots, x_{\sigma(n)}\right)
$$

is $\left(r_{\sigma(1)}, \ldots, r_{\sigma(n)}\right)$-directional shift stable with constant $k$.

Directional shift stability can also be seen as a weakening of linearity. The next important result is presented below.

Theorem 2. Let $F \in \mathcal{B}_{n}$. Then, $F$ is $\vec{r}$-directional shift stable for any directional vector $\vec{r}$ if and only if $F$ is an afinne function, i.e., $F(\mathbf{x})=u+\sum_{i=1}^{n} k_{i} x_{i}$ for some real constants $u, k_{1}, \ldots, k_{n}$.

Proof. Suppose $F(\mathbf{x})=u+\sum_{i=1}^{n} k_{i} x_{i}$. Then, for any $\vec{r}=\left(r_{1}, \ldots, r_{n}\right)$ it holds

$$
F(\mathbf{x}+c \vec{r})=u+\sum_{i=1}^{n} k_{i}\left(x_{i}+c r_{i}\right)=F(\mathbf{x})+k c,
$$

where $k=\sum_{i=1}^{n} k_{i} r_{i}$. Due to Theorem $1, F$ is $\vec{r}$-directionally shift stable.

Concerning the necessity, it is enough to consider that $F$ is $\vec{e}_{i}$-directionally shift stable, where $\vec{e}_{i}=(0, \ldots \underbrace{1, \ldots}, 0), i=1, \ldots, n$.

$i$ th coordinate 
Let $F\left(\mathbf{x}+c \vec{e}_{i}\right)=F(\mathbf{x})+k_{i} c$ for any $\mathbf{x} \in[0,1]^{n}$ and $c \in \mathbb{R}$ such that $\mathbf{x}+c \vec{e}_{i} \in$ $[0,1]^{n}$. Then

$$
\begin{aligned}
F(\mathbf{x}) & =F\left(\mathbf{0}+x_{1} \vec{e}_{1}+\cdots+x_{n} \vec{e}_{n}\right)=F\left(\mathbf{0}+x_{1} \vec{e}_{1}+\cdots+x_{n-1} \vec{e}_{n-1}\right)+k_{n} x_{n} \\
& =\cdots=F(\mathbf{0})+\sum_{i=1}^{n} k_{i} x_{i}
\end{aligned}
$$

proving the desired result with $u=F(\mathbf{0})$.

Observe that a linear function $F \in \mathcal{B}_{n}$ given by $F(\mathbf{x})=u+\sum_{i=1}^{n} k_{i} x_{i}$ satisfies:

- $\quad F \in \mathcal{F}_{n}$ whenever $u+\sum_{i=1}^{n} \min \left(k_{i}, 0\right) \geq 0$ and $u+\sum_{i=1}^{n} \max \left(0, k_{i}\right) \leq 1$;

- if $u=0, k_{1}, \ldots, k_{n} \geq 0$ and $\sum_{i=1}^{n} k_{i}=1$, then $F$ is a weighted arithmetic mean and $F \in \mathcal{A}_{n}$ (thus $F \in \mathcal{W}_{n}, F \in \mathcal{P}_{n}$ and $F \in \mathcal{S}_{n}$ ). Hence, this $F$ is a semi-aggregation function if and only if it is an aggregation function.

\section{Binary Shift-Invariant Functions}

Shift-invariant (difference scale invariant) functions were studied and characterized in several works; see, e.g., [4,10-13] (Chapter 7).

Definition 2. Let $F \in \mathcal{B}_{n}$. Function $F$ is deemed shift invariant whenever

$$
F\left(x_{1}+c, \ldots, x_{n}+c\right)=F\left(x_{1}, \ldots, x_{n}\right)+c,
$$

for any $\left(x_{1}, \ldots, x_{n}\right) \in[0,1]^{n}, c \in[0,1]$ and $\left(x_{1}+c, \ldots, x_{n}+c\right) \in[0,1]^{n}$.

When considering the shift invariantness, we deal with directional vector $\vec{r}=\overrightarrow{\mathbf{1}}=$ $(1, \ldots, 1)$, i.e., $F \stackrel{\text { s.s. }}{\sim}(\overrightarrow{\mathbf{1}}, 1)$.

For the sake of transparency, hereon, we fix $n=2$, i.e., we deal with binary functions only. We introduce an equivalence relation $\sim$ on $[0,1]^{2}$ by $(x, y) \sim(u, v)$ if and only if

$$
(x, y)=(u, v)+c(1,1) \quad \text { for some } c \in \mathbb{R},
$$

i.e., $(x-u, y-v)$ and $(1,1)$ are linearly dependent vectors. Obviously, the set of all equivalence classes of $\sim$ forms a partition of $[0,1]^{2}$. Consider a set $\mathcal{H}$ of single representative points of all such equivalence classes. Then, an arbitrary shift-invariant function $F \in \mathcal{B}_{2}$ is determined by its value in points from $\mathcal{H}$. Indeed, knowing $F(u, v)$ for any $(u, v) \in \mathcal{H}$, for each $(x, y) \in[0,1]^{2}$, there is $(z, t) \in \mathcal{H}$ such that $(x, y) \sim(z, t)$, and then $F(x, y)=$ $F(z, t)+x-z$.

We introduce three typical sets $\mathcal{H}$ :

- $\mathcal{H}_{1}=\left\{(u, v) \in[0,1]^{2} \mid \min (u, v)=0\right\}$;

- $\mathcal{H}_{2}=\left\{(u, v) \in[0,1]^{2} \mid \max (u, v)=1\right\}$;

- $\mathcal{H}_{3}=\left\{(u, v) \in[0,1]^{2} \mid u+v=1\right\}$.

These sets are depicted in the Figure 1. 

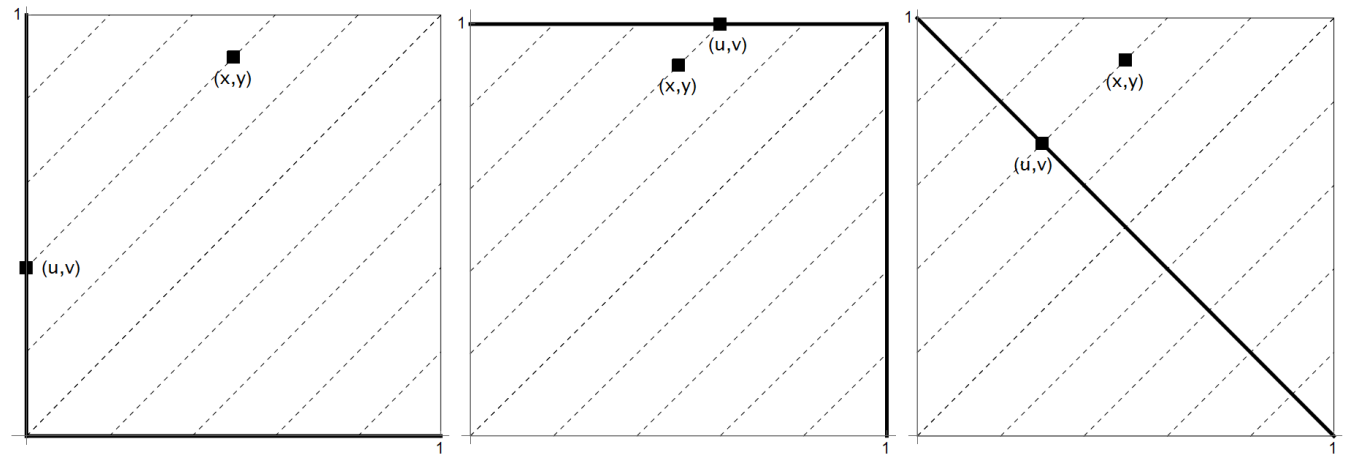

Figure 1. Bold lines correspond to the sets $\mathcal{H}_{1}$ (left), $\mathcal{H}_{2}$ (middle), and $\mathcal{H}_{3}$ (right).

The usual approach to characterize binary shift-invariant functions is related to $\mathcal{H}_{1}$. Then, introducing two unary functions $f, g:[0,1] \rightarrow \mathbb{R}, f(x)=F(x, 0)$ and $g(y)=F(0, y)$, $f(0)=g(0)=F(0,0), F \in \mathcal{B}_{2}$ is given by

$$
F(x, y)= \begin{cases}f(x-y)+y & \text { if } x \geq y \\ g(y-x)+x & \text { otherwise. }\end{cases}
$$

The only constraint on $f, g$ to generate $F \in \mathcal{B}_{2}$ by (3) is $f(0)=g(0)$ and the boundedness of $f$ and $g$. We summarize the properties of $f, g$ yielding $F$ from the remaining classes of the above-introduced functions. Note that for shift-invariant functions, fusion functions, semi-aggregation functions, pre-aggregation functions, and weak aggregation functions coincide.

Proposition 1. Let $F \in \mathcal{B}_{2}$ be shift invariant and given by (3). Then,

(i) $F \in \mathcal{F}_{2}\left(F \in \mathcal{S}_{2}, F \in \mathcal{P}_{2}, F \in \mathcal{W}_{2}\right)$ if and only if $0 \leq f(x) \leq x$ and $0 \leq g(y) \leq y$ for all $x, y \in[0,1]$;

(ii) $F \in \mathcal{A}_{2}$ if and only if both $f$, $g$ are non-decreasing and 1-Lipschitz (i.e., $\left|f\left(x_{1}\right)-f\left(x_{2}\right)\right| \leq$ $\left.\left|x_{1}-x_{2}\right|,\left|g\left(y_{1}\right)-g\left(y_{2}\right)\right| \leq\left|y_{1}-y_{2}\right|\right)$, and $f(0)=g(0)=0$.

Proof. Part (ii) can be found in [13].

Concerning (i), for any $(x, y) \sim(u, 0), F(x, y) \in[f(u), f(u)+1-u]$ and, for each $v \in[f(u), f(u)+1-u], v=F(x, y)$ for some $(x, y) \sim(u, 0)$. Suppose $F \in \mathcal{F}_{2}$. Then, necessarily $[f(u), f(u)+1-u] \subseteq[0,1]$, i.e., $0 \leq f(u)$ and $f(u)+1-u \leq 1$. Hence, $0 \leq f(u) \leq u$. Similarly, $0 \leq g(y) \leq y$ can be shown.

Vice-versa, suppose $F$ is given by (3) and $0 \leq f(x) \leq x$ and $0 \leq g(y) \leq y$ for any $x, y \in[0,1]$. Then,

- $\quad$ If $x \geq y, F(x, y)=f(x-y)+y \in[y, x] \subseteq[0,1]$.

- Similarly, if $x<y, F(x, y)=g(y-x)+x \in[x, y] \subseteq[0,1]$.

Summarizing, $F(x, y) \in[0,1]$ for any $(x, y) \in[0,1]^{2}$ and, thus, $F \in \mathcal{F}_{2}$.

When considering the set $\mathcal{H}_{2}$ to study binary shift-invariant functions, we have to consider functions $h, q:[0,1] \rightarrow \mathbb{R}, h(x)=F(x, 1)$ and $q(y)=F(1, y)$. Using similar arguments as in the previous case (when we have considered the set $\mathcal{H}_{1}$ ), for any $F \in \mathcal{B}_{2}$ it holds

$$
F(x, y)= \begin{cases}h(x+1-y)+y-1 & \text { if } x \geq y \\ q(y+1-x)+x-1 & \text { otherwise. }\end{cases}
$$

This $F$ belongs to $\mathcal{F}_{2}$ if and only if $x \leq h(x) \leq 1$ and $y \leq q(y) \leq 1$ for all $x, y \in[0,1]$. Moreover, $F$ belongs to $\mathcal{S}_{2}\left(\mathcal{P}_{2}, \mathcal{W}_{2}\right)$ if and only if $F \in \mathcal{F}_{2}$. Finally, this $F$ belongs to $\mathcal{A}_{2}$ (i.e., it is a shift-invariant binary aggregation function) if and only if $h(1)=g(1)=1$ and both $h$ and $q$ are non-decreasing and 1-Lipschitz. Note also that, if $F \in \mathcal{B}_{2}$, necessarily, 


$$
h(x)=g(1-x)+x \text { and } q(y)=f(1-y)+y \text { for all } x, y \in[0,1]
$$

Finally, when considering the set $\mathcal{H}_{3}=\{(x, 1-x) \mid x \in[0,1]\}$, for each $F \in \mathcal{B}_{2}$ it holds

$$
F(x, 1-x)= \begin{cases}g(1-2 x)+x & \text { if } x \leq \frac{1}{2} \\ f(2 x-1)+1-x & \text { otherwise }\end{cases}
$$

Let $t:[0,1] \rightarrow \mathbb{R}$ be given by $t(x)=F(x, 1-x)$. Due to (5), we can derive that

$$
F(x, y)=t\left(\frac{1+x-y}{2}\right)+\frac{x+y-1}{2} \text { for } x \in[0,1] .
$$

Note that there is, in general, no constraint on $t$ (clearly, up to the boundedness of $t$ ) to determine $F$ by Formula (6). Our $f$ belongs to $\mathcal{F}_{2}$ if and only if $\min (x, 1-x) \leq$ $t(x) \leq \max (x, 1-x)$ for all $x \in[0,1]$, (note that, then, necessarily, $\left.t\left(\frac{1}{2}\right)=\frac{1}{2}\right)$. Concerning the belongingness of our $F$ to $\mathcal{S}_{2}, \mathcal{P}_{2}$ or $\mathcal{W}_{2}$, it is equivalent to $F \in \mathcal{F}_{2}$. Finally, $F$ is a shift-invariant binary aggregation function if and only if $t\left(\frac{1}{2}\right)=\frac{1}{2}$ and $t$ is 1-Lipschitz.

Example 2. Let us consider the set $\mathcal{H}_{3}$ and let the function $t:[0,1] \rightarrow[0,1]$ bedefined as

$$
t(x)= \begin{cases}x^{2}+\frac{1}{4} & \text { if } x \leq \frac{1}{2} \\ 2 x-x^{2}-\frac{1}{4} & \text { else }\end{cases}
$$

Then, the corresponding function $F:[0,1]^{2} \rightarrow \mathbb{R}$ can be expressed by

$$
F(x, y)=\left\{\begin{array}{ll}
x+\left(\frac{x-y}{2}\right)^{2} & \text { if } x \leq y \\
x-\left(\frac{x-y}{2}\right)^{2} & \text { else }
\end{array} .\right.
$$

The functions $t(x)$ and $F(x, y)$ are illustrated in Figure 2.
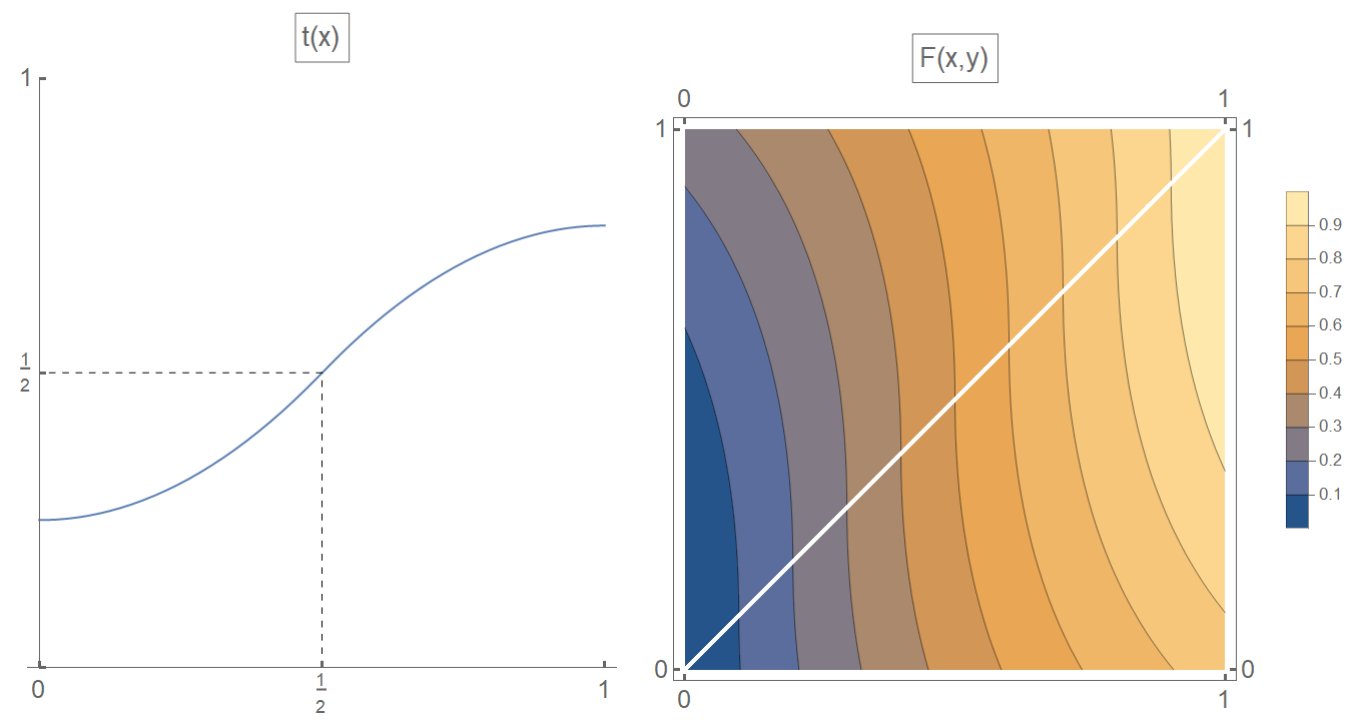

Figure 2. The generator $t(x)$ (on the left) and the corresponding function $F(x, y)$ (on the right) from Example 2.

Observe that we have no monotonicity constraint concerning $t$ generating a shiftinvariant binary aggregation function. The greatest possible $t$ is given by $t(x)=\max (x, 1-x)$ and then $F(x, y)=\max (x, y)$. The smallest $t$ is given by $t(x)=\min (x, 1-x)$ and then 
$F(x, y)=\min (x, y)$. Finally, if $t$ is constant $t(x)=\frac{1}{2}$ for all $x \in[0,1]$, then $F(x, y)=\frac{x+y}{2}$; i.e., $F$ is the arithmetic mean. Observe also that if $t$ is linear, $t(x)=c x+(1-c) \frac{1}{2}, c \in[-1,1]$, then $F$ is the weighted arithmetic mean, $F(x, y)=\lambda x+(1-\lambda) y$, where $\lambda=\frac{1+c}{2}$.

\section{General Binary Directional Shift-Stable Functions}

Based on representative sets $\mathcal{H}_{1}, \mathcal{H}_{2}$, or $\mathcal{H}_{3}$ (or their subsets, in dependence of $\vec{r}$ ), for any $\vec{r}$ and $k$, one can find $F \in \mathcal{B}_{2}$ such that $F$ is $\vec{r}$-directional shift stable with constant $k$, i.e., $F \stackrel{\text { s.s. }}{\sim}(\vec{r}, k)$. This is no longer the case if $F$ belongs to the above-considered proper subclasses of $\mathcal{B}_{2}$. Thus, for example, if $\vec{r}=(1,1)$, and both $F(0,0)$ and $F(1,1)$ belong to $[0,1]$ (clearly, this is the case if $F \in \mathcal{F}_{2}$ ), then

$$
F(1,1)=F((0,0)+1 \cdot \vec{r})=F(0,0)+k \cdot 1,
$$

and, thus, $k=F(1,1)-F(0,0) \in[-1,1]$. Moreover, if $F(0,0)=0$ and $F(1,1)=1$ (this is surely the case if $\left.F \in \mathcal{S}_{2}\right), k=1$ is the only possible value for $k$ once $F$ is $\vec{r}$-directional shift stable with $\vec{r}=(1,1)$.

Due to Formula (2), if, for a given $\vec{r} \neq(0,0)$, there is a constant $k \in \mathbb{R}$ such that $F \in \mathcal{B}_{2}$ and $F \stackrel{\text { s.s. }}{\sim}(\vec{r}, k)$, for any $\alpha \neq 0, F \stackrel{\text { s.s. }}{\sim}(\alpha \vec{r}, \alpha k)$. Then, to determine all possible nonzero constants $k$ such that there exist $F$ from some of discussed classes of functions, which $F \stackrel{\text { s.S. }}{\sim}(\vec{r}, k)$, it is enough to consider vectors $\vec{r}=\left(r_{1}, r_{2}\right)$ such that $\max \left(r_{1}, r_{2}\right)=1$. For such vectors, and for a subclass $\mathcal{C}$ of functions from $\mathcal{B}_{2}$, we denote by $K_{\vec{r}, \mathcal{C}}$ the set of all constants $k$ such that there is $F \in \mathcal{C}$ satisfying $F \stackrel{\text { s.s. }}{\sim}(\vec{r}, k)$. As previously mentioned, $K_{(1,1), \mathcal{B}_{2}}=\mathbb{R}$.

Additionally, $K_{(1,1), \mathcal{F}_{2}} \subseteq[-1,1]$. Consider $F_{1}=\max , F_{-1}=1-\max$, and for $k \in$ $[-1,1], F_{k}=\frac{k+1}{2} F_{1}+\frac{1-k}{2} F_{-1}=\frac{1-k}{2}+k \max$. Then

$$
F_{k}(\mathbf{x}+c(1,1))=\frac{1-k}{2}+k \max \left(x_{1}+c, x_{2}+c\right)=F_{k}(\mathbf{x})+k c,
$$

i.e., $F_{k} \stackrel{\text { s.s. }}{\sim}((1,1), k)$. Thus $K_{(1,1), \mathcal{F}_{2}}=[-1,1]$.

For the remaining discussed function classes, we have $K_{(1,1), \mathcal{S}_{2}}=K_{(1,1), \mathcal{P}_{2}}=K_{(1,1), \mathcal{W}_{2}}=$ $K_{(1,1), \mathcal{A}_{2}}=\{1\}$, and, thus, the related functions are shift invariant, and they are discussed in the previous section.

Similarly, for any $a \in[0,1]$, it holds $K_{(a, 1), \mathcal{F}_{2}}=K_{(1, a), \mathcal{F}_{2}}=[-1,1]$. Now, we discuss some particular directional vectors $\vec{r}$. Let $\vec{r}=\left(\frac{1}{2}, 1\right)$ and suppose $F \in \mathcal{S}_{2}$ such that $F \stackrel{\text { s.s. }}{\sim}(\vec{r}, k)$. Then, necessarily, $F$ also belongs to $\mathcal{P}_{2}$ due to the fact that

$$
0 \leq F\left(\frac{1}{2}, 0\right)=F\left((1,1)-\left(\frac{1}{2}, 1\right)\right)=1-k \leq 1
$$

and, hence, $k \geq 0$. Consider the set $\mathcal{H}_{1}$ and related functions $f$ and $g, f(x)=F(x, 0)$ and $g(y)=F(0, y)$. Then, $F \stackrel{\text { s.s. }}{\sim}(\vec{r}, k)$ means that

$$
F(x, y)= \begin{cases}f\left(x-\frac{y}{2}\right)+k y & \text { if } 2 x \geq y \\ g(y-2 x)+2 k x & \text { otherwise. }\end{cases}
$$

Necessarily, $f(0)=g(0)=0$ and $f\left(\frac{1}{2}\right)=1-k$, for any $0 \leq k \leq 1$. Note that, then, $k \geq 0$ means that $F \in \mathcal{S}_{2}$.

Define $F_{0}, F_{1} \in \mathcal{F}_{2}$ by

$$
F_{1}(x, y)=\operatorname{med}(2 x-1,2 x, y) \text { and } F_{0}(x, y)= \begin{cases}0 & \text { if } y \geq 2 x \\ 1 & \text { if } y \leq 2 x-1 \\ \frac{1}{2} & \text { otherwise }\end{cases}
$$


Then, $F_{1} \in \mathcal{A}_{2}, F_{1} \stackrel{\text { s.s. }}{\sim}(\vec{r}, 1)$ and $F_{0} \in \mathcal{P}_{2}, F_{0} \stackrel{\text { s.s. }}{\sim}(\vec{r}, 0)$. Then, for any $k \in[0,1], F_{k}=$ $k F_{1}+(1-k) F_{0} \in \mathcal{P}_{2}$ and $F_{k} \stackrel{\text { s.s. }}{\sim}(\vec{r}, k)$. Thus, $K_{\left(\frac{1}{2}, 1\right), \mathcal{S}_{2}}=K_{\left(\frac{1}{2}, 1\right), \mathcal{P}_{2}}=[0,1]$.

Moreover, if we suppose $F$ to be a binary aggregation function, necessarily, $F\left(\frac{1}{2}, 0\right)=$ $1-k \leq F\left(\frac{1}{2}, 1\right)=k$, i.e., $k \geq \frac{1}{2}$. It is not difficult to check that $G \in \mathcal{A}_{2}$ given by $G(x, y)=\operatorname{med}\left(x, \frac{y}{2}, \frac{1+y}{2}\right)$ satisfies $G \stackrel{\text { s.s. }}{\sim}\left(\vec{r}, \frac{1}{2}\right)$, which proves $K_{\left(\frac{1}{2}, 1\right), \mathcal{A}_{2}}=\left[\frac{1}{2}, 1\right]$.

Now, we characterize all $\left(\frac{1}{2}, 1\right)$-directional shift-stable binary aggregation functions, considering Formula (7).

Proposition 2. Let $F \in \mathcal{B}_{2}$ be given by Formula (7). Then, $F$ is a $\left(\frac{1}{2}, 1\right)$-directional shift-stable binary aggregation function if and only if there is $k \in\left[\frac{1}{2}, 1\right]$ such that $f(0)=0, f\left(\frac{1}{2}\right)=1-k, f$ is non-decreasing and $2 k$-Lipschitz, and $g(0)=0, g$ is non-decreasing and $k$-Lipschitz.

Proof. The proof follows from the previously shown fact that $K_{\left(\frac{1}{2}, 1\right), \mathcal{A}_{2}}=\left[\frac{1}{2}, 1\right]$, and showing the monotonicity of $F$ given by (7) in the first coordinate and in the second coordinate. For example, the constraint of $k$-Lipschitzianity of $g$ follows from the necessity of non-decreasingness of $g$ (i.e., of $F(0, \cdot))$ and the inequality $F(x+\epsilon, y) \geq F(x, y)$ valid for any $\epsilon>0$ such that $2(x+\epsilon) \geq y(0 \leq x \leq x+\epsilon \leq 1)$. Then,

$$
F(x+\epsilon, y)=g(y-2 x-2 \epsilon)+2 k(x+\epsilon) \geq g(y-2 x)+2 k x=F(x, y)
$$

means that

$$
\frac{g(y-2 x)-g(y-2 x-2 \epsilon)}{2 \epsilon} \leq k,
$$

i.e., $g$ is $k$-Lipschitz. Similarly, for appropriate $x, y, \epsilon$ we have

$$
f\left(x-\frac{y+\epsilon}{2}\right)+k(y+\epsilon) \geq f\left(x-\frac{y}{2}\right)+k y,
$$

i.e.,

$$
\frac{f\left(x-\frac{y}{2}\right)-f\left(x-\frac{y}{2}-\frac{\epsilon}{2}\right)}{\frac{\epsilon}{2}} \leq 2 k,
$$

showing the $2 k$-Lipschitzianity of $f$. The sufficiency is a matter of an easy processing and is thus omitted.

Example 3. Consider $k=\frac{3}{4}$ and define $f, g:[0,1] \rightarrow[0,1]$ by $f(x)=\frac{x}{2}$ and $g(y)=\frac{3}{4} y$. Then, $f, g$ satisfy the constraints of Proposition 2 and, applying (7), we obtain the aggregation function $A \stackrel{\text { s.S. }}{\sim}\left(\left(\frac{1}{2}, 1\right), \frac{3}{4}\right)$, given by

$$
A(x, y)=\max \left(\frac{x+y}{2}, \frac{3 y}{4}\right) \text {. }
$$

Using similar argument as we have considered in the case $\vec{r}=\left(\frac{1}{2}, 1\right)$, the next results can be shown.

Proposition 3. Let $a \in[0,1]$ and $\vec{r}=(a, 1), \vec{s}=(1, a)$. Then,

- $K_{\vec{r}, \mathcal{B}_{2}}=K_{\vec{s}, \mathcal{B}_{2}}=\mathbb{R}$;

- $\quad K_{\vec{r}, \mathcal{F}_{2}}=K_{\vec{s}, \mathcal{F}_{2}}=[-1,1]$;

- $\quad K_{\vec{r}, \mathcal{S}_{2}}=K_{\vec{r}, \mathcal{P}_{2}}=K_{\vec{s}, \mathcal{S}_{2}}=K_{\vec{s}, \mathcal{P}_{2}}=[0,1]$;

- $\quad K_{\vec{r}, \mathcal{A}_{2}}=K_{\vec{s}, \mathcal{A}_{2}}=[a, 1]$.

Proposition 4. Let $a \in[0,1]$ and $\vec{r}=(a, 1)$. For $F \in \mathcal{B}_{2}$, denote $f(x)=F(x, 0)$ and $g(y)=$ $F(0, y)$. 
(i) If $a=0$, then $F \stackrel{\text { s.s. }}{\sim}(\vec{r}, k), k \in \mathbb{R}$ if and only if

$$
F(x, y)=f(x)+k y,
$$

and, then, $F$ is an aggregation function if and only if $f(0)=0, f$ is non-decreasing, $k \in[0,1]$ and $f(1)=1-k$.

(ii) If $a>0$, then $F \stackrel{\text { s.s. }}{\sim}(\vec{r}, k), k \in \mathbb{R}$ if and only if

$$
F(x, y)= \begin{cases}f(x-a y)+k y & \text { if } x \geq a y \\ g\left(y-\frac{x}{a}\right)+\frac{k}{a} x & \text { otherwise }\end{cases}
$$

and then $F$ is an aggregation function if and only if $f(0)=g(0)=0, f, g$ are non-decreasing, $k \in[a, 1], f(1-a)=1-k, f$ is $\frac{k}{a}$-Lipschitz and $g$ is $k$-Lipschitz.

Note that results for directional vectors $(1, a)$ are the same; we only need to exchange the corresponding functions $f$ and $g$. Therefore, for example, based on Example 3, we see that $A(x, y)=\max \left(\frac{x+y}{2}, \frac{3 x}{4}\right)$ is an aggregation function $A \stackrel{\text { s.s. }}{\sim}\left(\left(1, \frac{1}{2}\right), \frac{3}{4}\right)$.

For any $\vec{r}=(a, 1)(\vec{s}=(1, a))$ with $a \in[0,1]$, we can fix the constant $k \in[a, 1]$ and find related extremal $\vec{r}(\vec{s})$-directional shift-stable aggregation functions related to $k$. Obviously, these extremal aggregation functions are related to extremal possible functions $f$ and $g$. Based on the constraints given in Proposition 4, the next interesting result is presented below.

Proposition 5. Under the constraints of Proposition 4, fix $k \in[a, 1]$. Then,

(i) If $a=0$ and $F \in \mathcal{A}_{2}$, then $F$ is the greatest (smallest) aggregation function $F \stackrel{\text { s.s. }}{\sim}((0,1), k)$ if it is determined by $f^{*}\left(f_{*}\right)$ given by

$$
f^{*}(x)=\left\{\begin{array} { l l } 
{ 0 } & { \text { if } x = 0 , } \\
{ 1 - k } & { \text { if } x \in ] 0 , 1 ] , }
\end{array} \quad \left(f_{*}(x)=\left\{\begin{array}{ll}
0 & \text { if } x \in[0,1[, \\
1-k & \text { if } x=1
\end{array}\right),\right.\right.
$$

and, then, it is given by

$$
\begin{gathered}
F(x, y)=\left(A_{k, 0}\right)^{*}(x, y)= \begin{cases}k y & \text { if } x=0, \\
1-k+k y & \text { otherwise, }\end{cases} \\
\left(F(x, y)=\left(A_{k, 0}\right)_{*}(x, y)=\left\{\begin{array}{ll}
k y & \text { if } x<1, \\
1-k+k y & \text { otherwise }
\end{array}\right) .\right.
\end{gathered}
$$

Both families $\left(\left(A_{k, 0}\right)^{*}\right)_{k \in[0,1]}$ and $\left(\left(A_{k, 0}\right)_{*}\right)_{k \in[0,1]}$ are pairwise incomparable, i.e., if $k_{1} \neq k_{2}$, then $\left(A_{k_{1}, 0}\right)^{*}$ and $\left(A_{k_{2}, 0}\right)^{*}\left(\left(A_{k_{1}, 0}\right)_{*}\right.$ and $\left.\left(A_{k_{2}, 0}\right)_{*}\right)$ are incomparable aggregation functions.

(ii) If $a>0$ and $F \in \mathcal{A}_{2}$, then $F=\left(A_{k, a}\right)^{*}$ is the greatest $\left(F=\left(A_{k, a}\right)_{*}\right.$ is the smallest) aggregation function $F \stackrel{\text { s.s. }}{\sim}((a, 1), k)$ if it is determined by $f^{*}$ and $g^{*}$ given by

$$
f^{*}(x)=\operatorname{med}\left(\frac{k}{a} x, 1-k, \frac{k}{a} x+1-\frac{k}{a}\right) \text { and } \quad g^{*}(y)=k y
$$

(by $f_{*}$ and $g_{*}$ given by

$$
\left.f_{*}(x)=\operatorname{med}\left(0,1-k, \frac{k}{a} x+1-\frac{k}{a}\right) \text { and } \quad g_{*}(y)=0\right),
$$


and then it is given by

$$
\begin{gathered}
\left(A_{k, a}\right)^{*}(x, y)= \begin{cases}k y & \text { if } x-a y \leq 0, \\
\frac{k}{a} x & \text { if } 0<x-a y \leq \frac{a}{k}-a, \\
1-k+k y & \text { if } \frac{a}{k}-a<x-a y \leq 1-a, \\
\frac{k}{a} x+1-\frac{k}{a} & \text { otherwise, }\end{cases} \\
\left(A_{k, a}\right)_{*}(x, y)=\left\{\begin{array}{ll}
\frac{k}{a} x & \text { if } x-a y \leq 0, \\
k y & \text { if } 0<x-a y \leq 1-\frac{a}{k}, \\
\frac{k}{a} x+1-\frac{k}{a} & \text { if } 1-\frac{a}{k}<x-a y \leq 1-a, \\
1-k+k y & \text { otherwise }
\end{array}\right),
\end{gathered}
$$

see Figure 3. For any fixed $a \in] 0,1]$, both families $\left(\left(A_{k, a}\right)^{*}\right)_{k \in[a, 1]}$ and $\left(\left(A_{k, a}\right)_{*}\right)_{k \in[a, 1]}$ are pairwise incomparable.
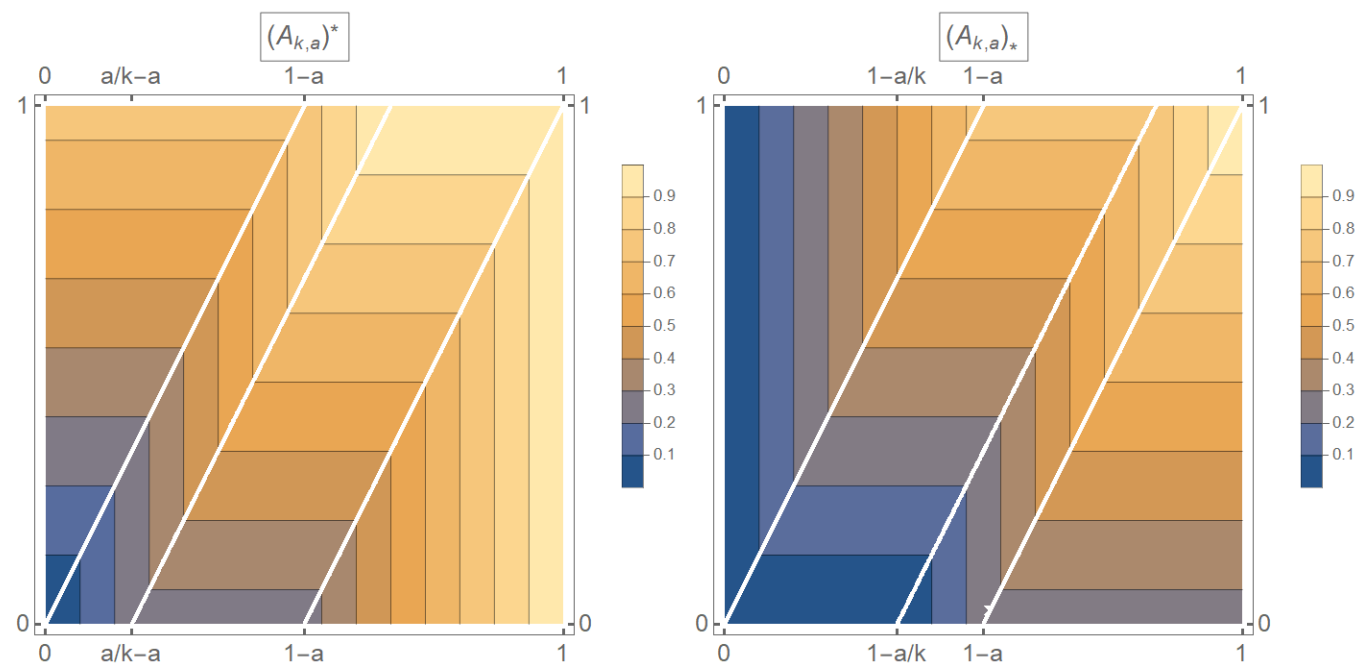

Figure 3. The greatest (on the left) and the smallest (on the right) aggregation functions $\left(A_{k, a}\right)^{*}$ and $\left(A_{k, a}\right)_{*}$.

\section{Conclusions}

Inspired by generalization of monotone functions into directionally monotone functions and shift invariantness (difference scale invariantness) of $n$-ary real functions defined on $[0,1]^{n}$, we introduced a new notion of directional shift-stable functions. This new type of stability is related to a non-zero directional $n$-ary vector $\vec{r}$ and a real constant $k$ expressing the output increment of a function $F$ once the increment of its argument $\mathbf{x}$ is just $\vec{r}$, and proportionally if the argument $\mathbf{x}$ is just $\vec{r}$ or if the argument increment is $c \cdot \vec{r}, F(\mathbf{x}+c \vec{r})=F(\mathbf{x})+k c$. Observe that the class of $\vec{r}$-directional shift-stable aggregation (pre-aggregation) functions does not change if we replace the vector $\vec{r}$ by a vector $u . \vec{r}$; here, $u$ is an arbitrary positive real constant. This fact allows us to fix the considered directional vectors $\vec{r}$ by the constrain $\max \left(r_{1}, \ldots, r_{n}\right)=1$, as exemplified in Section 4 . As an important advantage of $\vec{r}$-shift-invariant aggregation functions, one can consider the fact that instead of their description on the full domain $[0,1]^{n}$, it is enough to have them fixed on a significantly smaller subdomain. This fact is stressed in Section 3; see the subdomains $\mathcal{H}$ exemplified there. Our approach can also be seen as a special generalization of the linearity of functions. Indeed, if $F:[0,1]^{n} \rightarrow \mathbb{R}$ is an $\vec{r}$-directional shift-stable function, then, for any points $\mathbf{x}, \mathbf{y} \in[0,1]^{n}$ such that vectors $\mathbf{x}-\mathbf{y}$ and $\vec{r}$ are linearly dependent, $F$ is linear on the segment determined by $\mathbf{x}$ and $\mathbf{y}$.

Although this paper is purely theoretical, we expect to see applications of our results in measurement theory but also in image processing, classification systems, and related domains where directional monotonicity is successfully considered; see, e.g., [8,14,15]. 
Note that for any successful application, a proper choice of considered theoretical tools is necessary. This fact opens several new problems for the future study of our approach. In particular, based on the real data, we aim to focus on fitting appropriate directional vector $\vec{r}$ to be fixed. Based on our theoretical results, we can then deduce possible values of constants $k$ characterizing the increments of $\vec{r}$-directionally shift-stable functions. Fixing the direction $\vec{r}$ and knowing the possible domain for constants $k$, another task is the appropriate determination of the constant $k$.

Author Contributions: Formal analysis, A.S. and R.M.; methodology, R.M.; project administration, A.S.; resources, A.S. and R.M.; supervision, R.M.; writing—review and editing, A.S. Both authors have read and agreed to the published version of the manuscript.

Funding: The support of the grants APVV-17-0066, VEGA 1/0468/20, and also the Palacky University Olomouc IGAPrF2021 is gratefully announced.

Institutional Review Board Statement: Not applicable.

Informed Consent Statement: Not applicable.

Acknowledgments: The authors are thankful to the anonymous referees for giving valuable comments and suggestions which helped to improve the final version of this paper.

Conflicts of Interest: The authors declare no conflict of interest.

\section{References}

1. Beliakov, G.; Bustince Sola, H.; Calvo, T. A Practical Guide to Averaging Functions; Studies in Fuzziness and Soft Computing 329; Springer: Cham, Switzerland, 2016; ISBN 978-3-319-24751-9.

2. Beliakov, G.; Pradera, A.; Calvo, T. Aggregation Functions: A Guide for Practitioners; Springer: Heidelberg, Germany, 2007.

3. Gagolewski, M. Data Fusion: Theory, Methods, and Applications; Institute of Computer Science, Polish Academy of Sciences: Warszawa, Poland, 2015; ISBN 978-83-63159-20-7.

4. Grabisch, G.; Marichal, J.-L.; Mesiar, R.; Pap, E. Aggregation Functions; Cambridge University Press: Cambridge, UK, 2009.

5. Torra, V.; Narukawa, Y. Modeling Decisions: Information Fusion and Aggregation Operators; Springer: Berlin/Heidelberg, Germany; New York, NY, USA, 2007; ISBN 978-3-540-68789-4.

6. Wilkin, T.; Beliakov, G. Weakly monotone aggregation functions. Int. J. Intell. Syst. 2016, 30, 144-165. [CrossRef]

7. Bustince, H.; Fernández, J.; Kolesárová, A.; Mesiar, R. Directional monotonicity of fusion functions. Eur. J. Oper. Res. 2015, 244, 300-308. [CrossRef]

8. Lucca, G.; Sanz, J.A.; Dimuro, G.P.; Bedregal, B.; Mesiar, R.; Kolesárová, A.; Bustince, H. Pre-aggregation functions: Construction and an application. IEEE Trans. Fuzzy Syst. 2016, 24, 260-272. [CrossRef]

9. Kuczma, M. An Introduction to the Theory of Functional Equations and Inequalities. Cauchy's Equation and Jensen's Inequality; Birkhäuser: Basel, Switzerland, 2009; ISBN 978-3-7643-8748-8.

10. Aczél, J.; Gronau, D.; Schwaigen, J. Increasing solutions of the homogeneity equation and of similar equations. J. Math. Anal. Appl. 1994, 182, 436-464. [CrossRef]

11. Lucce, R.D.; Krantz, D.H.; Supes, P.; Tversky, A. Foundations of Measurement, Vol. III: Representation, Axiomatization, and Invariance; Academic Press Inc.: San Diego, CA, USA, 1990.

12. Roberts, F.S. Measurement Theory, with Applications to Decision-Making, Utility and the Social Sciences, Volume 7 of Encyclopedia of Mathematics and Its Applications; Addison-Wesley Publishing Co.: Reading, MA, USA, 1979.

13. Lázaro, J.; Rückschlossová, T.; Calvo, T. Shift invariant binary aggregation operators. Fuzzy Sets Syst. 2004, 142, 51-62. [CrossRef]

14. Lucca, G.; Sanz, J.A.; Dimuro, G.P.; Bedregal, B.; Bustince, H.; Mesiar, R. CF-integrals: A new family of pre-aggregation functions with application to fuzzy rule-based classification systems. Inf. Sci. 2018, 435, 94-110. [CrossRef]

15. Wieczynski, J.C.; Dimuro, G.P.; Borges, E.N.; Santos, H.S.; Lucca, G.; Lourenzutti, R.; Bustince, H. Generalizing the GMC-RTOPSIS Method using CT-integral Pre-aggregation Functions. In Proceedings of the 2020 IEEE International Conference on Fuzzy Systems (FUZZ-IEEE), Glasgow, UK, 19-24 July 2020; pp. 1-8. 